\title{
A Mechanism for How a Particle Detects and Moves in a Field Gradient
}

\author{
Dirk J. Pons \\ Department of Mechanical Engineering, University of Canterbury, Christchurch, New Zealand \\ Email:dirk.pons@canterbury.ac.nz
}

How to cite this paper: Pons, D.J. (2020) A Mechanism for How a Particle Detects and Moves in a Field Gradient. Journal of Modern Physics, 11, 1560-1575. https://doi.org/10.4236/jmp.2020.1110097

Received: September 1, 2020

Accepted: October 13, 2020

Published: October 16, 2020

Copyright $\odot 2020$ by author(s) and Scientific Research Publishing Inc. This work is licensed under the Creative Commons Attribution International License (CC BY 4.0).

http://creativecommons.org/licenses/by/4.0/

\begin{abstract}
Problem: The mechanisms whereby force operates are poorly understood at the fundamental level. Purpose: This paper proposes a mechanism for how a particle detects the field gradient, and how it moves therein. Approach: A non-local hidden-variable (NLHV) theory is used, specifically the Cordus NLHV theory. Findings: The operation of force is proposed to occur from the interaction between the energisation sequence of the particle, with the field gradient, resulting in discrete displacement motions of the particle. Specifically the particle sub-structures sweep through a volume of space during their energisation cycle. This locus is warped by the incoming field, hence preferentially displacing the particle along the gradient. Originality: The novel contribution of this work is providing a candidate mechanism for how a particle detects and moves in a field gradient.
\end{abstract}

\section{Keywords}

Field, Interaction, Fundamental, Quantum

\section{Introduction}

The operation of force is something taken for granted. It is intuitively accepted that a particle moves in the gradient of a field. However given that a particle is also assumed to be a zero-dimensional (0-D) point, it is unclear how such a particle would detect the direction or gradient of the field.

A common, though simplistic, explanation is by analogy to a small sphere (e.g. a marble) on an inclined plane in a gravitational situation: it will naturally roll downhill. However this analogy does not extend to a 0-D point. For the marble, the line of action of the centre of mass is offset from the contact point with the surface, so a moment arises to roll the ball downwards. For a 0-D point there cannot be a moment arm, hence the analogy fails. If a particle did have 
volume, either by a volumetrically dispersed aggregation of other $0-\mathrm{D}$ particles (the quantum mechanics premise), or because fundamental particles had volume (the premise of hidden variable theories), it is unclear how that volume might help the particle detect the field direction in real situations outside of the analogy.

The slope analogy represents the field as continuous. Another explanation might be attempted by assuming it is discrete, i.e. mediated by the exchange of gauge bosons. In that case it is easier to understand that there may be more incoming gauge bosons on one side of a test particle than on the other, and that this underlies the field gradient. However what such theories fail to explain is the direction of force. What is the mechanism that causes opposite electrical charges to attract, and similar to repel? How does an incoming gauge boson cause movement of the $0-\mathrm{D}$ test particle?

Hence there is an underlying ontological problem with describing how field force interactions occur at the level of fundamental particles. This paper proposes a mechanism for how a particle detects the field gradient, and how it moves therein.

\section{Context}

The explanation was developed from a non-local hidden-variable (NLHV) theory, specifically the Cordus theory. All NLHV theories propose that fundamental particles have sub-structure, though differ greatly in what they propose for those structures. In the case of the Cordus theory the proposal is for an open string-like structure. The structure was inferred by applying design principles to the photon behaviour of the double-slit device, and thereby determining what set of physical features would be sufficient to explain the observations [1]. The outcome was a particle with two reactive ends connected by a fibril. The fibril is inert relative to other particles and provides the coordination between the two ends. This design intrinsically accommodates non-local behaviour. The reactive ends are periodically energized at the de Broglie frequency, during which they emit discrete forces into space in three orthogonal emission directions, see Figure 1. These directions are denoted $r$, a, and $t$ in the figure [2] [3]. The particle interacts with other particles only at its reactive ends. The discrete forces are joined into flux tubes. Since these propagate out into space, the space between particles therefore comprises a fabric of discrete forces [4].

The key conceptual point of departure is the proposal that a particle has two separate ends. In contrast, conventional theories have the particle being a zero dimensional point, or a distribution of substance around a central point. In the Cordus theory, there is nothing at the nominal central location of the particle. The idea of two reactive ends is conceptually close to a string theory interpretation, and the dipole concept of classical electromagnetism. Indeed the number of parameters required to define the Cordus particle structure is similar to the number of dimensions in some string theories [6]. The theory describes multiple physical phenomena, see Table 1 . 
Table 1. Phenomena for which the Cordus theory has an explanation. Adapted from [7].

\begin{tabular}{|c|c|c|}
\hline Phenomenon explained & Key concept & Reference \\
\hline Wave-particle duality in the double slit device & One reactive end passes through each slit. & [1] \\
\hline Derivation of optical laws from a particle perspective & $\begin{array}{l}\text { Includes derivation of reflection and refraction laws, } \\
\text { and Brewster's Angle from particle basis. }\end{array}$ & [1] \\
\hline Prediction of particle structures & Electron, proton, neutron, neutrino species, photon. & $\begin{array}{c}{[5][8][9][10]} \\
{[11]}\end{array}$ \\
\hline $\begin{array}{l}\text { Explanation of the decay processes and prediction } \\
\text { of a deeper decay model }\end{array}$ & Dependency identified on neutrino species loading. & [9] [10] \\
\hline $\begin{array}{l}\text { Explanation for the selective spin characteristics of } \\
\text { neutrinos whereby the direction of spin is correlated } \\
\text { with the matter-antimatter species }\end{array}$ & $\begin{array}{l}\text { Spin direction arises from reaction between incomplete } \\
\text { discrete force emissions from the particle, } \\
\text { and the background fabric. }\end{array}$ & [10] \\
\hline $\begin{array}{l}\text { Explanation for particle spin and derivation } \\
\text { of the electron } \mathrm{g} \text { factor } \mathrm{g}=2\end{array}$ & Cordus particle structure naturally causes $\mathrm{g}=2$. & [7] \\
\hline Explanation for the annihilation process & $\begin{array}{l}\text { Description of the discrete force changes involved in } \\
\text { remanufacture of these particle identities. Includes a } \\
\text { conceptual explanation of the difference between otho- } \\
\text { and para-positronium decay rates (ortho and para } \\
\text { refer to spin combinations of the bound electron } \\
\text { and anti-electron/positron). }\end{array}$ & [12] \\
\hline Provision of a mechanics for pair production & $\begin{array}{l}\text { Rearrangement of discrete forces changes } \\
\text { the particle identity. }\end{array}$ & [13] \\
\hline Explanation of process of photon emission & $\begin{array}{l}\text { Excess energy in the electron changes it span, } \\
\text { which is opposed by bonding constraints. }\end{array}$ & [3] [14] \\
\hline Synchronous interaction & $\begin{array}{l}\text { Synchronous interaction between discrete forces of different } \\
\text { matter particles causes the strong nuclear force. }\end{array}$ & [2] \\
\hline $\begin{array}{l}\text { Predicted structure of atomic nuclei and explanation } \\
\text { of stability for nuclides } \mathrm{H} \text { to } \mathrm{Ne}\end{array}$ & $\begin{array}{l}\text { Protons and neutrons are arranged in a nuclear polymer. } \\
\text { The rules for this arrangement, and for the bridge neutrons, } \\
\text { are inferred and are qualitatively consistent with observed } \\
\text { stability/instability/non-existence of all nuclides in this range. }\end{array}$ & [15] [16] \\
\hline $\begin{array}{l}\text { Prediction of a mechanism for } \\
\text { asymmetrical baryogenesis }\end{array}$ & $\begin{array}{c}\text { Predicts a decay path for remanufacture of the antielectron } \\
\text { to the proton. This also solves the asymmetrical } \\
\text { leptogenesis problem. }\end{array}$ & [8] \\
\hline Origin of entropy & $\begin{array}{l}\text { Fabric increases the Irreversibility of geometric } \\
\text { position of particle. }\end{array}$ & [17] \\
\hline $\begin{array}{l}\text { A theory for time as an emergent property of } \\
\text { matter rather than a universal attribute }\end{array}$ & $\begin{array}{l}\text { Time arises from the interaction between the frequency } \\
\text { of a particle and the local density of the fabric. }\end{array}$ & [4] \\
\hline Nature of the vacuum and the cosmological horizon & $\begin{array}{l}\text { Vacuum comprises fabric of discrete forces from } \\
\text { massy particles. }\end{array}$ & [18] \\
\hline Origin of the finite speed of light & $\begin{array}{c}\text { Determined by fabric density, hence variable with epoch } \\
\text { of universe and local distribution of mass. }\end{array}$ & [19] \\
\hline $\begin{array}{l}\text { Quantitative derivation of the relativistic } \\
\text { Doppler and the Lorentz factor }\end{array}$ & $\begin{array}{l}\text { Derivation accomplished from a particle perspective. } \\
\text { Identifies fabric density as a covert variable. }\end{array}$ & [20] \\
\hline
\end{tabular}




\section{Electron e}

Characterised by one discrete force in each of the three directions. This balanced loading causes the structure to be stable against decay

Physical structure

The discrete forces are released rather than retained as in the photon. Consequently there is an enduring succession of discrete forces in each of the three directions, which creates a long-ranged force effect.

New discrete forces continue to be created and sent down the flux tube at each frequency cycle

Inner Fibril provides instantaneous communication between reactive ends, hence a non-local effect

Type of reactive end: pulsatile. One reactive end energising and the other de-energising $\left(180^{\circ}\right.$ out of phase)

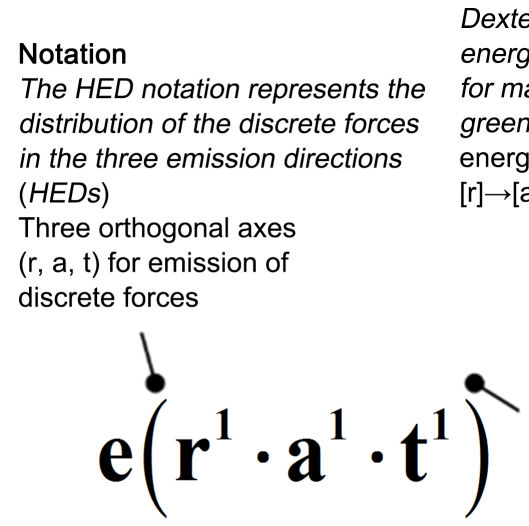

\section{Notation} (HEDs) $(r, a, t)$ for emission of

$$
\text { (11 }
$$

Figure 1. The representation of the electron's internal and external structures. It is proposed that the particle has three orthogonal discrete forces, energised in turn at each reactive end. Adapted from [5].

\section{Approach}

Prior work showed that the Lorentz, relativistic Doppler, and time dilation could be derived from first principles using the Cordus theory [20], but required the flux tube to be stretchable. This imposes a conceptual conundrum. How could discrete forces, which are required in the context of synchronous bonding [2] to be in a binary state of energisation, also be continuous as required in the general relativity context? The existence of this duality implies the potential existence of a deeper mechanics, once that could give either outcome depending on the perspective taken. The specific situation under examination was how the electro-magneto-gravitational fields operated, i.e. the long ranged interactions (ex- 
cluding the strong and weak).

The approach used abductive reasoning. It started with the high-level behaviour, and applied inference to determine the possible deeper mechanics. Conceptual propositions, or lemmas, were identified. These are indicated§.

\section{Results}

\subsection{A Proposed General Mechanism for Force}

Consider test particle B, say an electron, in an electrostatic field set up by particle A. Each particle has two reactive ends, and for $B$ these are denoted $B_{1}$ and $B_{2}$. See Figure 2. The location of interest is reactive end $B_{1}$ which receives forces from $A$, and emits its own discrete forces.

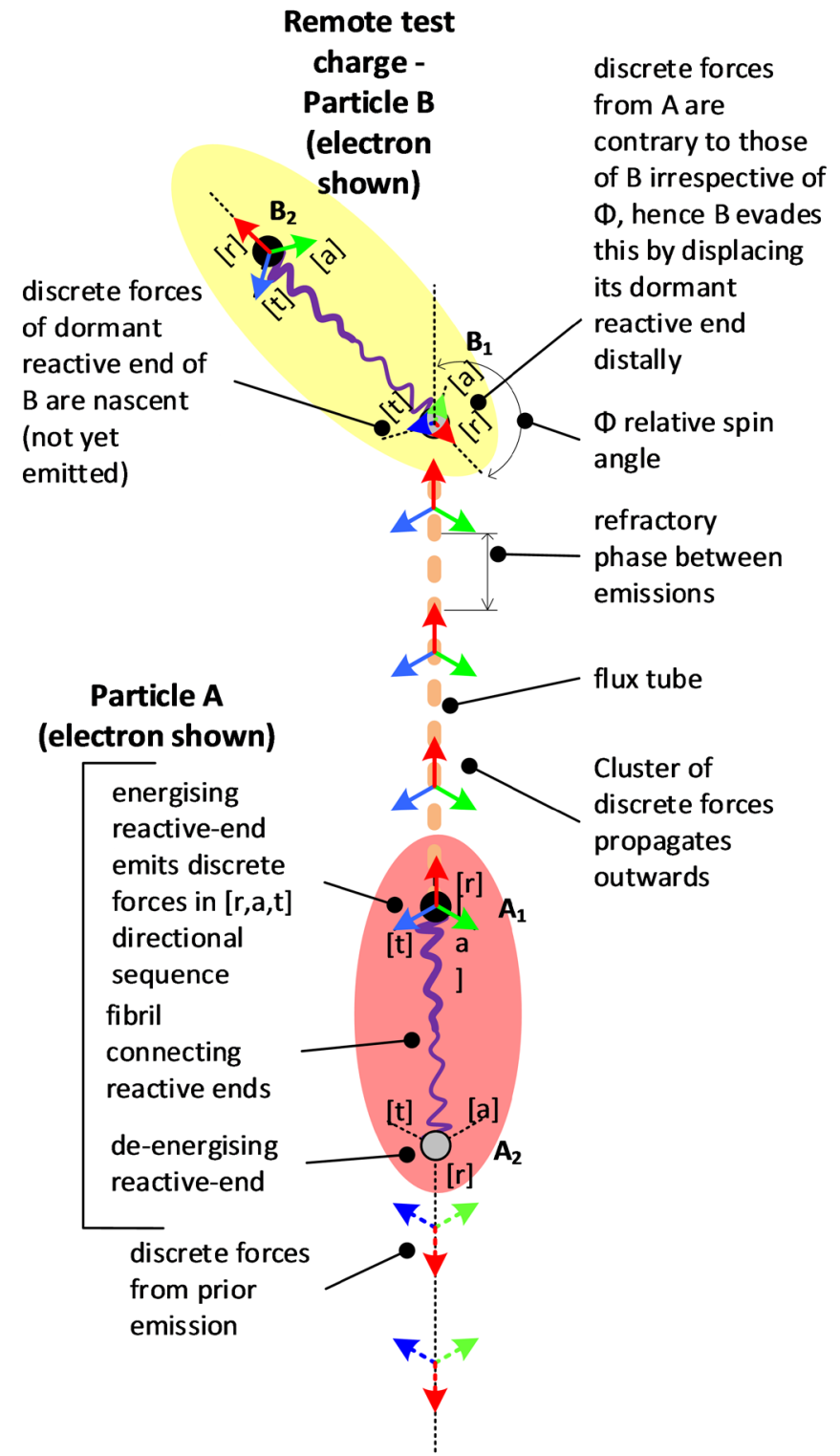

Figure 2. Discrete forces emitted by basal particle A, and intercepted by remote test particle B. Each particle has two reactive ends. 
Emission of discrete forces occurs at the energisation frequency of the particle (\$1)

It is proposed that discrete forces are emitted from massy particles, and at each cycle of energisation a fresh discrete force is created and emitted. It follows that higher frequency particles, i.e. those that are in more energetic states, emit discrete forces more frequently. The corollary is that the magnitude of the force effect is proposed to be determined by the number of discrete forces received.

Similar-charged particles interact via the transphasic synchronous interaction, while opposite-charged particles interact via the cisphasic interaction (\$2)

The theory predicts that bonds between proximal particles arise from synchronisation of their discrete force emissions. This is called the synchronous interaction [2] and has been applied to predict the structure of the atomic nucleus for multiple nuclides [15] [16], and also explains Pauli pair structures [7]. Favourable emission states have already been defined for this theory, see [2], and include assembly of opposite charge discrete forces and complete sets of discrete forces. There are two subtypes to the synchronous interaction, which are transphasic and cisphasic. These are when reactive ends from different particles energise out- and in-phase respectively.

This lemma extends the synchronous principle to ranged particles: it is proposed that the same mechanism applies even if the particles are some distance apart, e.g. in the electrostatic force and ranged forces generally. If the emission of discrete forces of one particle favours the emissions of the other, then the particles move closer together. The direction of motion is along the gradient in a direction that favours increased compatibility or evades incompatibility (as the case may be).

Force is caused by coercive displacement of reactive ends (\$3)

Under these assumptions, force at the deeper level is a process of discrete displacements of reactive ends, under the coercive effect of incoming discrete forces. More specifically, force on test particle B is caused by incoming discrete forces interacting with the emission sequence of B's discrete forces. This coerces the reactive end to re-energise in a different location. Those incoming discrete forces may be from other particle A or the fabric (many other particles) generally.

The nature of the interference for $B$ is the phase difference between its intended emissions vs. the incoming emissions. This prescription causes the reactive end of B to move its location in space, such that it more nearly synchronises its emissions in or out of phase (cis- or transphasic respectively) with the external discrete forces. The process is one of the reactive end of B being drawn to, or evading, the discrete forces produced by distant particles.

Such a motion is a finite displacement and occurs during the energisation cycle of the particle, hence we refer to it as a coerced discrete displacement. It is the particle's response to the external discrete force. This has the effect that the reactive end energises at a different location to its natural preference, hence 
causing the position of the particle as a whole to change, and this effect is perceived as force.

The response depends on the bound state of B. For free particle B the free reactive end may move in response to the external discrete forces. For a partially bonded particle $\mathrm{B}$, one reactive end is co-located with the reactive end of other particles, and hence $B$ is constrained in span and frequency and this limits the available locations into which it can move. For particle B bonded at both reactive ends, there is no ability to move. If there is sufficient reaction force applied to the test particle, then it can be prevented from making this discrete displacement. Instead the body as a whole responds to the external discrete forces. Hence a larger body is subject to the same effect of prescribed displacement, but in an aggregate manner. Thus the effect of prescribed displacement scales from the small to the macroscopic discoherent state. If the external discrete forces are sufficiently compelling, even full bonded particle A may be broken free from its assembly. This is consistent with the effect of ionising radiation.

The displacement is not uniform or fixed, being instead determined by the relative strengths of the native vs. incoming discrete force emissions (hence also type and mass of test particle), and the degree of freedom or constraint on the test particle. The test particle makes a small displacement each time it de-energises, and hence the underlying response to the field is a series of discrete motions. As particles have high frequencies (de Broglie frequency), this process is apparently continuous at the macroscopic scale. Hence the explanation does not undermine classical continuous mechanics, but rather offers a deeper explanation. Likewise the force bosons of quantum mechanics are re-explained as discrete forces. Quantum mechanics requires different bosons for each interaction, whereas the Cordus theory proposes that different attributes of the discrete forces result in the different interactions.

\section{A field comprises sequential discrete forces ( $\$ 4)$}

A field is interpreted to comprise discrete forces, the action of which causes a particle to move in a rapid series of finite displacements in space. The perturbing external discrete force is of finite duration, hence the displacement per energisation cycle is also finite. For a particle B in a steady field, the next discrete force it encounters causes a displacement consistent with the previous one. Hence the particle in this situation shows a consistent direction of discrete displacements, hence a motion of increasing velocity, i.e. acceleration in response to the force field. Thus the effect of the force appears smooth and continuous at the macroscopic level of examination, even though it is fundamentally discrete.

\section{Discrete forces are connected in flux tubes (\$5)}

The sequence of discrete forces emitted by any one particle is proposed to be connected in a flux tube, this being a curvilinear assembly of discrete forces. The discrete force in the flux tube is a persistent structure even when not energised [20]. As a particle moves, so the next discrete force is emitted from a different position of the reactive end. Hence the direction of action of the following dis- 
crete force will be slightly different to that before it. Thus the flux tube is expected to have bends, for a moving emitter.

The discrete force interactions are fundamentally unidirectional ( $\$ 6)$

It is proposed that the displacement effect of a received discrete force, and hence the force interaction, is fundamentally unidirectional: when test particle $\mathrm{B}$ receives a discrete force, it reacts to it via the displacement effect. The emitting particle $A$ is unaffected, unless it also receives a discrete force from $B$. This means that the process whereby A emits a discrete force does not commit A to a future interaction dependent on whether or not that discrete force is intercepted by $\mathrm{B}$.

This is an unorthodox premise since force is generally held to be reciprocal in action. Indeed in the type of situation in which the Earth is positioned, similar particles affect each other equally, and hence the bi-directional nature of force is recovered. Nonetheless in general the theory predicts unidirectional effects: discrete forces from A unidirectionally affect B, and those from B affect A, but there is no fixed reciprocity or an exchange per se.

Discrete forces are not consumed in the interactions (\$7)

Discrete forces are not consumed by interactions but continue to propagate outward to affect yet other particles. In specific cases, the discrete force may be consumed, such as annihilation of matter-antimatter particles [12], and pair-production [13].

The capability to make discrete displacement of a reactive end is affected by its energisation state ( $\$ 8)$

The discrete displacement of a reactive end is affected by its energisation state. A reactive end that is fully energised in one of its emission directions $(r, a, t)$ is momentarily stationary in that direction, or would prefer to be so. At times of incomplete energisation the reactive end is mobile and most susceptible to being displaced by an incoming discrete force.

The reactive end samples the discrete forces around it (\$9)

A reactive end experiences all the discrete forces passing through that region of space which it occupies in its migrations. The reactive end is transparent in that the external discrete forces intrude into the reactive end temporarily as they propagate. A particle is not solely affected by the fields (or in this case the discrete forces) passing through its centre (or in this case its two reactive ends). Instead it is affected by discrete forces in the nearby locality of the reactive end. The incoming discrete forces occupy a volume of space during their transit. Consequently the reactive end has the ability to sense what is happening in the volume of space immediately around it, with an interaction between the external environment, discrete forces, and reactive end. This is non-local behaviour.

Sampling of the environment provides the mechanism for the reactive end to determine the field gradient ( $\$ 10)$

At intermediate stages of energisation the reactive end moves a small distance in reaction as it undertakes its own emissions. This results in a small-scale repe- 
titive displacement motion of the reactive end at the de Broglie frequency. This motion is in all three directions of space, which allows the reactive end to sweep through-and sample-a small volume of space. This is proposed as the mechanism whereby it senses the gradient of the external field.

The mechanism whereby the reaction end moves under the influence of an external field arises because the RE spends longer time in those regions of space where the density (or strength) of external discrete forces is more favourable (higher or lower depending on relative attributes of particles A and B). It preferentially energises there. Since energisation also means the RE is stationary, it dwells there longer, hence the mean position of the RE is changed.

\subsection{Mechanism for Force $\&$ Discrete Movement in an Imposed Field}

Above it was stated that force is caused by coercive displacement of reactive ends $(\$ 3)$, that the reactive end samples the discrete forces around it $(\$ 9)$, and that this provides the mechanism for the reactive end to determine the field gradient $(\$ 10)$. These conceptual propositions are now represented in a quantitative formulation.

Discrete force emissions take the form $\sin ^{2}(\theta / 2) \quad(\$ 11)$

Previous diagrams have shown the reactive ends as either energised or not, but this is simplistic. The strength of energisation, hence also of the emitted discrete forces, evolves over time. The question is what the shape of this energisation function might be. We are required, for reasons of logical consistency with the Lorentz work [20], to see the reactive ends as producing a continuous flux tube, without breaks, so abrupt step-like functions are excluded. It is logical to assume it takes a sinusoidal function, and ranges between 1 and 0 over a cycle (discrete forces of massy particles do not change sign, though photons do). We also need to consider the second reactive end $B_{2}$ and that the energy oscillates between the two-this is an established principle of the theory. This requires that the total energy is conserved at any one moment. A relationship that fits these criteria is $\sin ^{2}(\theta / 2)$ where $\theta$ is the phase angle of energisation, and this is marked as a lemma.

Thus the potential energy $\mathrm{U}$ of a discrete force in a particular direction (say $\mathrm{r}$ ) at reactive ends $B_{1}$ and $B_{2}$ is:

$$
\begin{aligned}
U_{B_{1}} & =\sin ^{2} \frac{\theta_{B}}{2} \\
U_{B_{2}} & =\cos ^{2} \frac{\theta_{B}}{2}
\end{aligned}
$$

Given also that there are three orthogonal discrete force emissions ( $r, a, t)$ and that the energisation sequence of these determines the matter-antimatter species [12], then the potential energy is partitioned into three components offset at thirds of the phase cycle:

$$
U_{B_{1}}(r)=\sin ^{2} \frac{\theta_{B}}{2}
$$




$$
\begin{aligned}
& U_{B_{1}}(a)=\sin ^{2}\left(\frac{\theta_{B}}{2}-120^{\circ}\right) \\
& U_{B_{1}}(t)=\sin ^{2}\left(\frac{\theta_{B}}{2}-240^{\circ}\right)
\end{aligned}
$$

See Figure 3 for a representation of these three phases. The discrete force emissions do not subtract energy from the particle.

Thus the reactive end is never fully de-energised (except momentarily in one axis), and this is consistent with the expectations from the Lorentz derivation for a stretchable flux tube. This also means that the energy at the basal generator, i.e. the reactive end $B_{1}$ has a circular function, see Figure 4 .

Thus the phased emission of discrete forces corresponds to a torsional energisation that is carried out into space by the discrete forces. The remote particle $B$ receives this torsional package of discrete forces, and assuming both $\mathrm{A}$ and $\mathrm{B}$ are matter particles, finds this conducive to its own emissions and moves closer along the field gradient.

The process of emitting discrete forces causes the reactive end to move cyclically in the $(r, a, t)$ directions $(\$ 12)$

It is proposed that the sinusoidal potential energy function of the discrete forces corresponds to a movement of the reactive end itself. The nature of the motion is inferred as follows. The discrete forces themselves are a type of potential displacement. Hence the displacement that underpins them is the square root of their potential energy function, hence a $\sin (\theta / 2)$ dependency. However the motion must be cyclical, i.e. the reactive end must return to its original

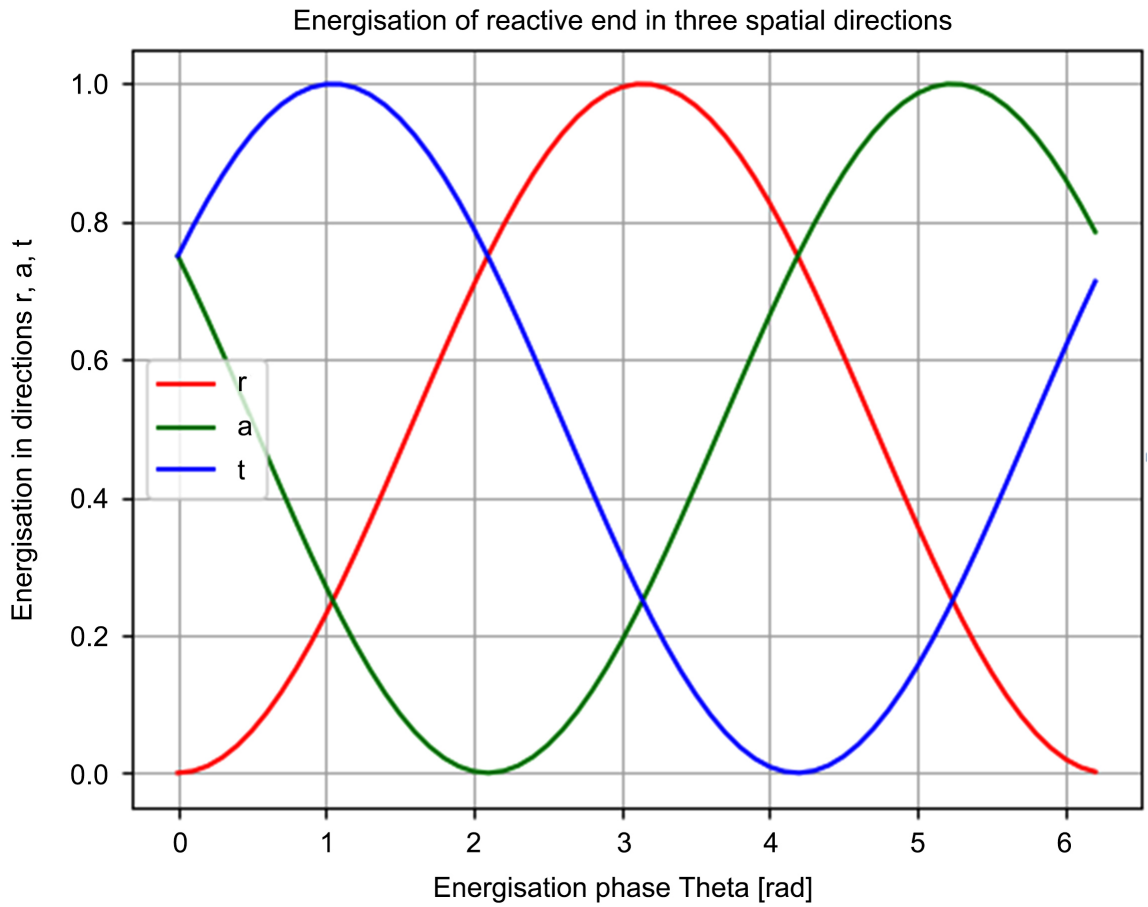

Figure 3. Energisation phases in the three orthogonal emission directions $(r, a, t)$ follow a $\sin ^{2}(\theta / 2)$ relation. 


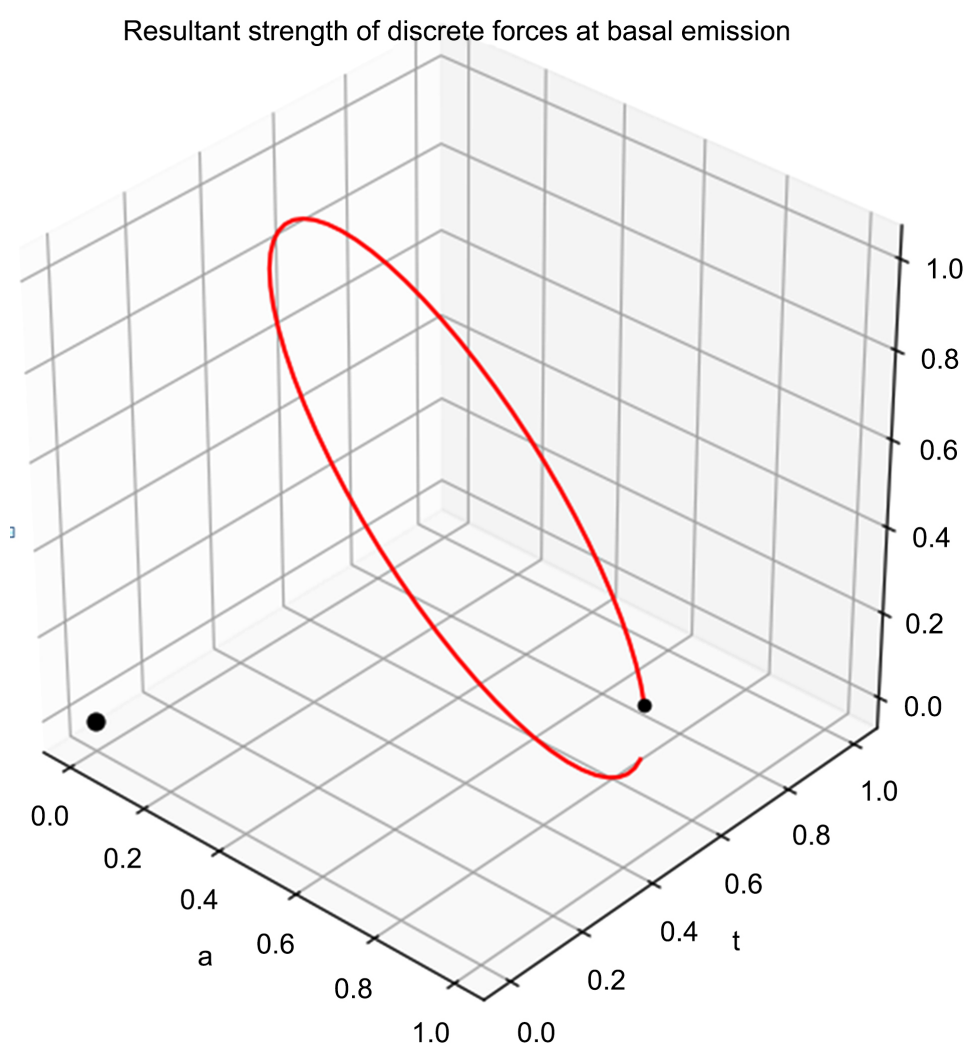

Figure 4. Resultant energy at the basal generator $B_{1}$ due to a matter negative charge. The axes are $(r, a, t)$. The larger black marker indicates the nominal origin $(0,0,0)$, and the smaller black marker indicates the location for $\theta=0$. The chart is deliberately shown incomplete to indicate the direction of rotation.

position, and this requires positive and negative components to its motion, hence a $\sin (\theta)$ function. Finally, the motion of the reactive end is inversely related to its energisation, being motionless when fully energised, hence a $\cos (\theta)$ relationship. This is shown for a single axis in Figure 5, and for all three axes in Figure 6. Thus the displacement of the reactive end may be inferred to be a circular locus around the nominal location of the reactive end.

While the positional locus is circular in the absence of an imposed field, the reactive end expands its excursion when in a field. Assuming a field gradient applies and consider only the $\mathrm{r}$ direction. The amount of displacement from the locus is presumed to be determined by two factors. The first is the strength of the field, which for simplicity is assumed to be linear (which is approximately true far from the field origin) with gradient $P_{\text {grad }}$ and strength $P_{\text {centre }}$ at the nominal centre point of the reactive end. Thus one side of the positional locus experiences the field as slightly stronger than the other. The second factor is the mobility of the reactive end, which is inversely related to its energisation, hence to $\cos ^{2} \theta / 2$. The result is a non-linear distortion of the positional locus of the form:

$$
r_{\text {moved }}(\theta)=r(\theta)+\left(P_{\text {grad }} r(\theta)+P_{\text {centre }}\right) \cos ^{2} \frac{\theta}{2}
$$


Locus of reactive end displacement [green], and Discrete force potential energy [red] in the $r$ direction

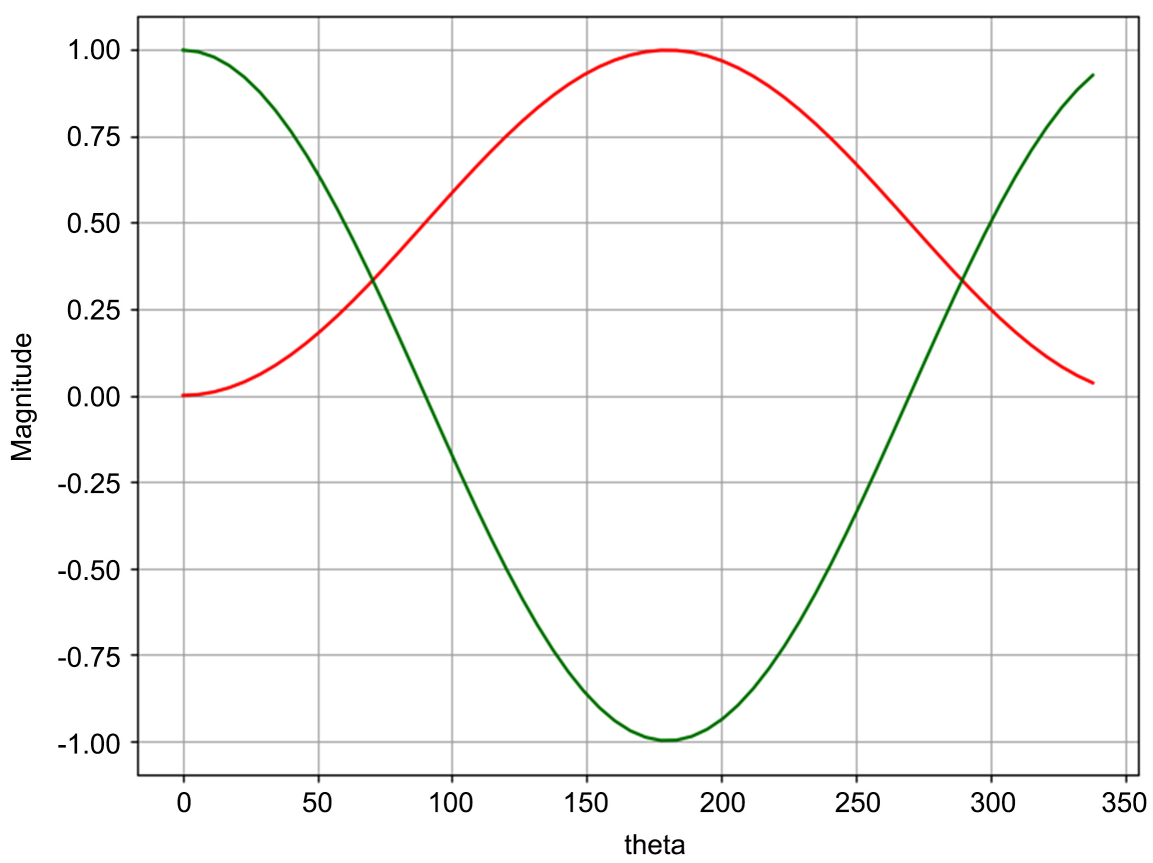

Figure 5. The locus of motion of the reactive end follows a $\cos (\theta)$ dependency for each axis, whereas the potential energy follows $\sin ^{2}(\theta / 2)$.

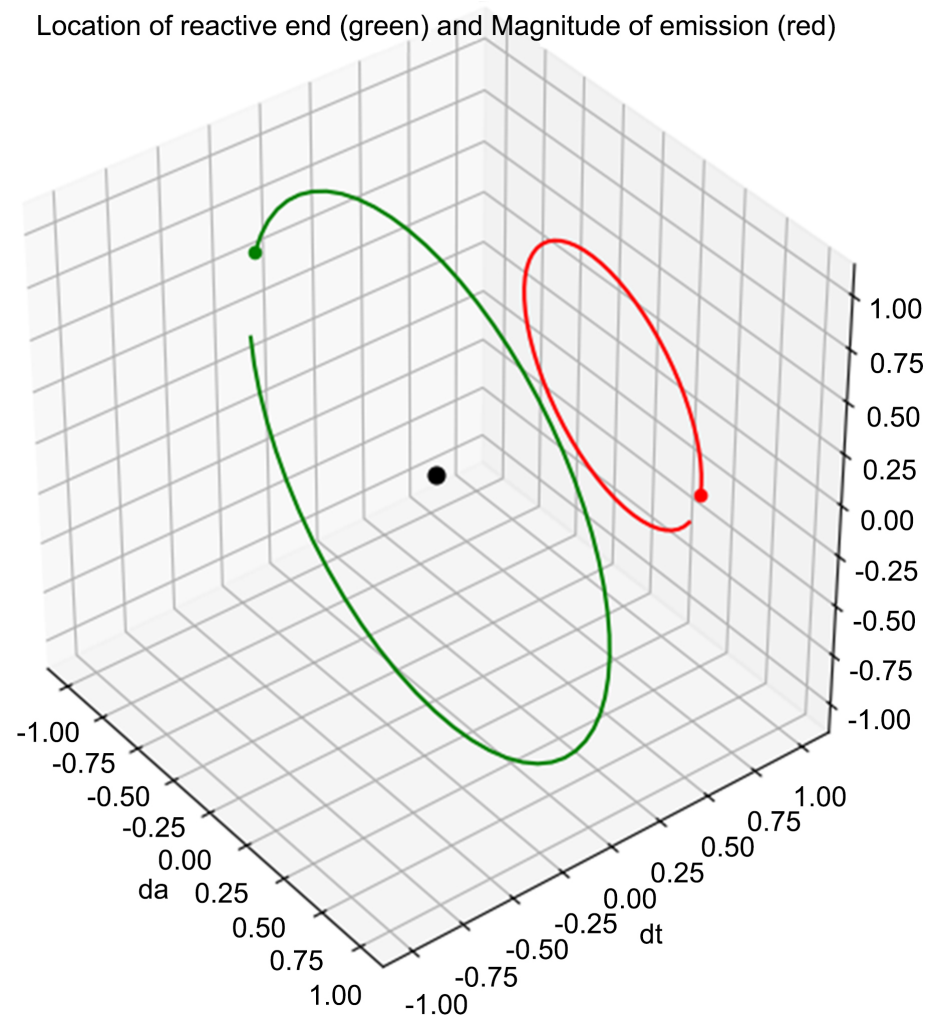

Figure 6. Circular locus of positon of the reactive end over a complete cycle of emission from both reactive ends, for an electron. Green shows the location locus, and red the energy. The vertical axis is the $r$ direction. 
An example of a distorted positional locus is shown in Figure 7(a). Even with a linear field the new locus is a non-linear distortion of the original circular track, i.e. the reactive end is sensitive to the spatial change in field strength. The progressive accumulation of displacement results in a distorted spiral locus with dwell regions of low progression, see Figure 7(b).

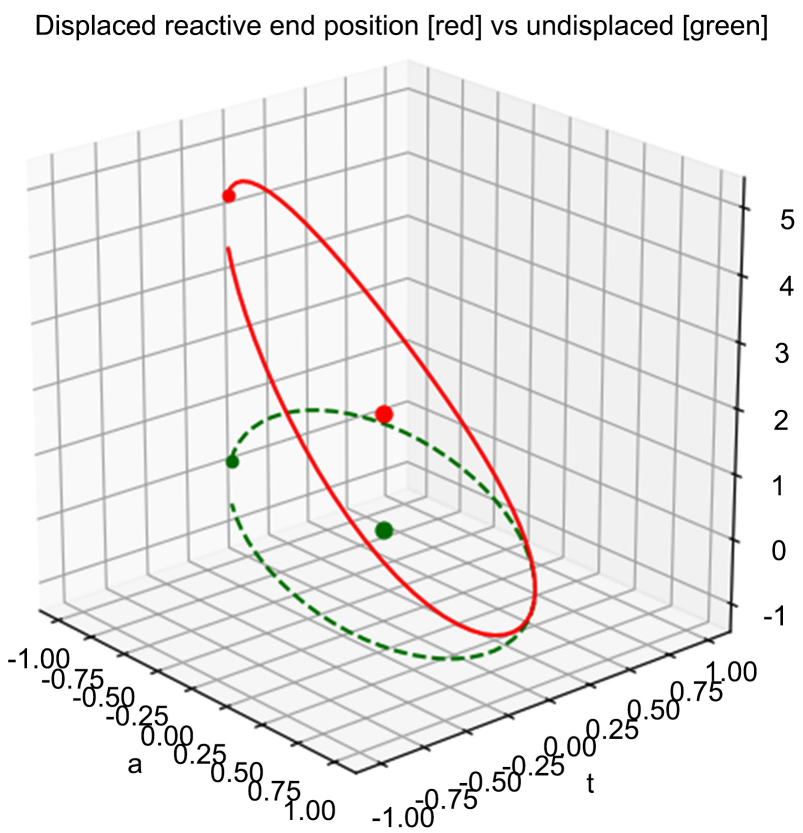

(a)

Cumulative displaced reactive end position [red] vs undisplaced [green]

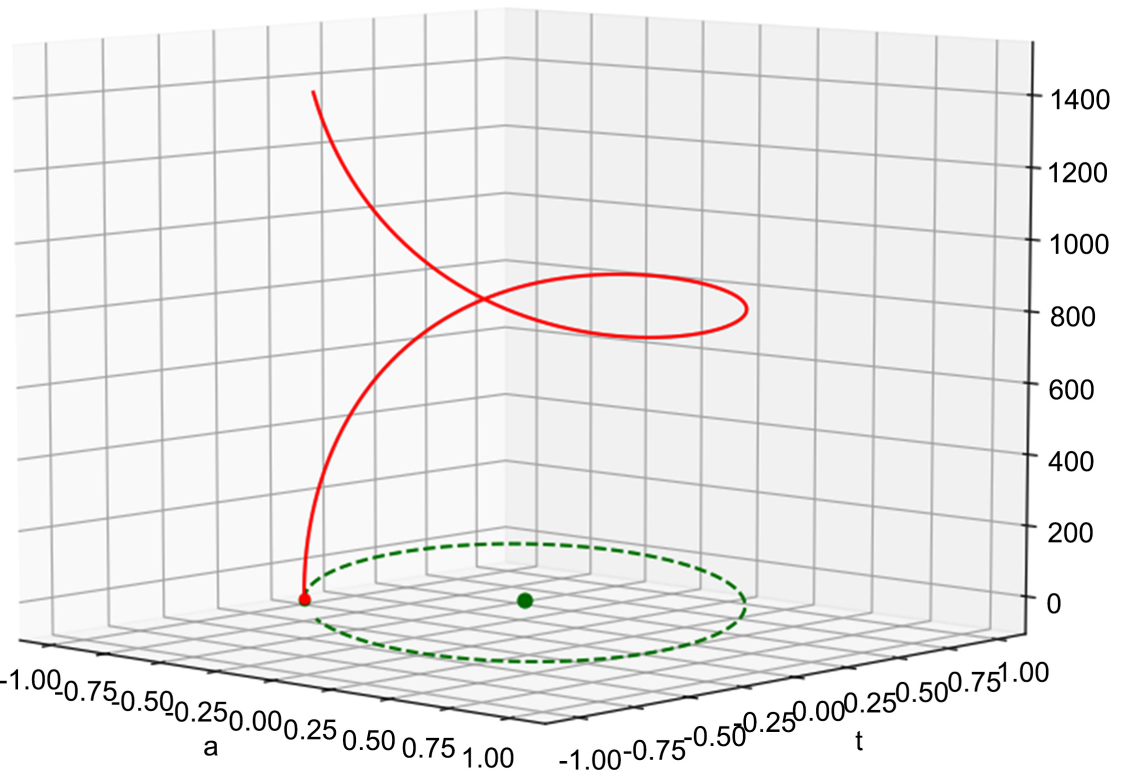

(b)

Figure 7. (a) Distorted positional locus for a reactive end under the effect of a linear field in the $r$ direction. The larger round symbols show the centre point of the locus, i.e. the nominal position of the reactive end. Values in the $r$ direction are nominal; (b) Cumulative locus of the reactive end. Values are nominal. 
The significance is that the mean location of the reactive end changes under the effect of the field. This adjustment occurs in discrete intervals at each frequency cycle while the reactive end is exposed to the field. Thus the following lemma is noted.

The positional locus of a reactive end is distorted by the effect of field gradient (\$13)

While the above example is based on an electrostatic interaction, there does not appear to be impediment to generalise the principles to any of the ranged fields.

\section{Discussion}

\subsection{Findings}

The novel contribution of this work is providing a candidate mechanism for how a particle detects and moves in a field gradient. The explanation accommodates the continuous vs. discrete duality of forces, as evidenced in the general relativity vs. quantum perspectives. Emitted discrete forces have a sinusoidal strength function. At the receiving particle, the mobility of the reactive end is inversely related to its energisation, so the interaction has dwell periods. The emitted field is continuous but the effect is discrete.

It also answers another duality question: how can a flux tube be continuous if the forces it contains are discrete [20]? The answer is that the reactive end binary states of energised vs. de-energised are an approximation of a sinusoidal strength relationship for energisation. Furthermore, with emissions in three orthogonal directions, there is no point in time when the flux tube is completely de-energised, hence its continuity is preserved.

\subsection{Limitations and Future Research Opportunities}

The present work has elucidated a mechanism for force generally. This has been formulated in a discrete force function. It could be interesting to develop specific formulations for each of the three ranged interactions: electrostatic, magnetic, and gravitational. There may different formulations for the discrete force function that could also be acceptable.

\section{Conclusions}

Per this theory, the operation of force at a more fundamental level is proposed to occur via the following mechanisms.

1) The reactive end moves in a cyclic locus in reaction to its own emission of discrete forces, hence sweeps through a volume of space during its energisation cycle.

2) This motion provides a mechanism for the reactive end to sample the field gradient around it.

3) The locus is warped by the discrete forces of the incoming field, hence preferentially displacing the reactive end along the gradient. The mechanism is that 
the reactive end spends longer time in those regions of space where the density (or strength) of external discrete forces is more favourable, and this changes its mean position in space. This adjustment occurs in discrete intervals at each frequency cycle while the reactive end is exposed to the field.

4) The resulting direction of motion is along the gradient in a direction that favours increased compatibility or evades incompatibility between the discrete forces from the two particles.

The underlying mechanism for force is therefore proposed to be a process of discrete displacements of reactive ends, under the coercive effect of incoming discrete forces from the field.

\section{Conflict of Interest Statement}

The author declares that there are no financial conflicts of interest regarding this work.

\section{References}

[1] Pons, D.J., et al. (2012) Physics Essays, 25, 132-140. https://doi.org/10.4006/0836-1398-25.1.132

[2] Pons, D.J., Pons, A.D. and Pons, A.J. (2013) Applied Physics Research, 5, 107-126. https://doi.org/10.5539/apr.v5n5p107

[3] Pons, D.J., Pons, A.D. and Pons, A.J. (2016) Journal of Modern Physics, 7, 1049-1067. https://doi.org/10.4236/jmp.2016.710094

[4] Pons, D.J., Pons, A.D. and Pons, A.J. (2013) Applied Physics Research, 5, 23-47. https://doi.org/10.5539/apr.v5n6p23

[5] Pons, D.J. (2015) Internal Structure of the Electron (Image Licence Creative Commons Attribution 4.0). Wikimedia Commons, (Creative Commons Attribution 4.0 International License).

[6] Pons, D.J. (2012) A Physical Interpretation of String Theory? http://vixra.org/abs/1204.0047

[7] Pons, D.J., Pons, A.D. and Pons, A.J. (2019) Journal of Modern Physics, 10, 835-860. https://doi.org/10.4236/jmp.2019.107056

[8] Pons, D.J., Pons, A.D. and Pons, A.J. (2014) Journal of Modern Physics, 5, 1980-1994. https://doi.org/10.4236/jmp.2014.517193

[9] Pons, D.J., Pons, A.D. and Pons, A.J. (2015) Applied Physics Research, 7, 1-11. https://doi.org/10.5539/apr.v7n1p1

[10] Pons, D.J., Pons, A.D. and Pons, A.J. (2014) Applied Physics Research, 6, 50-63. https://doi.org/10.5539/apr.v6n3p50

[11] Pons, D.J. (2015) Internal Structure of the Photon (Image Licence Creative Commons Attribution 4.0). Wikimedia Commons, (Creative Commons Attribution 4.0 International License).

[12] Pons, D.J., Pons, A.D. and Pons, A.J. (2014) Applied Physics Research, 6, 28-46. https://doi.org/10.5539/apr.v6n2p28

[13] Pons, D.J., Pons, A.D. and Pons, A.J. (2015) Journal of Nuclear and Particle Physics, 5, 58-69.

[14] Pons, D.J. (2015) Applied Physics Research, 7, 14-26. 
https://doi.org/10.5539/apr.v7n4p24

[15] Pons, D.J., Pons, A.D. and Pons, A.J. (2015) Physics Research International, 2015, Article ID: 651361. https://doi.org/10.1155/2015/651361

[16] Pons, D.J., Pons, A.D. and Pons, A.J. (2013) Applied Physics Research, 5, 145-174. https://doi.org/10.5539/apr.v5n6p145

[17] Pons, D.J., Pons, A.D. and Pons, A.J. (2016) Journal of Modern Physics, 7, 1277-1295. https://doi.org/10.4236/jmp.2016.710113

[18] Pons, D.J. and Pons, A.D. (2013) The Open Astronomy Journal, 6, 77-89. https://doi.org/10.2174/1874381101306010077

[19] Pons, D.J., Pons, A.D. and Pons, A.J. (2016) Applied Physics Research, 8, 111-121. https://doi.org/10.5539/apr.v8n3p111

[20] Pons, D.J., Pons, A.D. and Pons, A.J. (2018) Journal of Modern Physics, 9, 500-523. https://doi.org/10.4236/jmp.2018.93035 